EPJ Web of Conferences 106, 03003 (2016)

DOI: $10.1051 /$ epjconf/201610603003

(C) Owned by the authors, published by EDP Sciences, 2016

\title{
Analysis of dpa Rates in the HFIR Reactor Vessel using a Hybrid Monte Carlo/Deterministic Method ${ }^{\mathrm{a}}$
}

\author{
J.M. Risner ${ }^{1, \mathrm{~b}}$ and E.D. Blakeman (retired) \\ Oak Ridge National Laboratory, P.O. Box 2008, MS-6170, Oak Ridge, TN 37831, USA
}

\begin{abstract}
The Oak Ridge High Flux Isotope Reactor (HFIR), which began full-power operation in 1966, provides one of the highest steady-state neutron flux levels of any research reactor in the world. An ongoing vessel integrity analysis program to assess radiation-induced embrittlement of the HFIR reactor vessel requires the calculation of neutron and gamma displacements per atom (dpa), particularly at locations near the beam tube nozzles, where radiation streaming effects are most pronounced. In this study we apply the Forward-Weighted Consistent Adjoint Driven Importance Sampling (FW-CADIS) technique in the ADVANTG code to develop variance reduction parameters for use in the MCNP radiation transport code. We initially evaluated dpa rates for dosimetry capsule locations, regions in the vicinity of the HB-2 beamline, and the vessel beltline region. We then extended the study to provide dpa rate maps using three-dimensional cylindrical mesh tallies that extend from approximately $12 \mathrm{in}$. below to approximately $12 \mathrm{in}$. above the height of the core. The mesh tally structures contain over 15,000 mesh cells, providing a detailed spatial map of neutron and photon dpa rates at all locations of interest. Relative errors in the mesh tally cells are typically less than $1 \%$.
\end{abstract}

\section{Background}

The Oak Ridge High Flux Isotope Reactor (HFIR), which began full-power operation in 1966, provides one of the highest steady-state neutron flux levels of any research reactor in the world. While the original primary purpose of HFIR was the production of transuranic isotopes, its main mission has transitioned to neutron scattering research. HFIR provides four horizontal beam tubes (referred to as HB-1 through HB-4) for neutron scattering studies. HB-2 was substantially enlarged in 2000/2001, and a liquid hydrogen cold neutron source was installed in HB-4 in 2006/2007. An ongoing vessel integrity analysis program to assess radiation-induced embrittlement of the HFIR reactor vessel requires the calculation of neutron and gamma displacements per atom (dpa), particularly at locations near the beam tube nozzles, where radiation streaming effects are most pronounced.

\footnotetext{
a Notice: This manuscript has been authored by UT-Battelle, LLC, under Contract No. DE-AC0500OR22725 with the US Department of Energy. The US Government retains and the publisher, by accepting the article for publication, acknowledges that the US Government retains a nonexclusive, paid-up, irrevocable, worldwide license to publish or reproduce the published form of this manuscript, or allow others to do so, for the US Government purposes.

${ }^{\mathrm{b}}$ Corresponding author: risnerjm@ornl.gov
}

This is an Open Access article distributed under the terms of the Creative Commons Attribution License 2.0, which permits unrestricted use, distribution, and reproduction in any medium, provided the original work is properly cited. 
Previous analyses of neutron and photon dpa rates [1, 2] for HFIR were generally performed using a combination of two-dimensional (2D) and three-dimensional (3D) discrete ordinates calculations. While that methodology was reasonable for the relatively small set of dpa locations that were analyzed for many years, it was limited by the discretization errors that affect the accuracy that can be obtained using a deterministic analysis (i.e., the approximations inherent in the use of spatial mesh, discrete ordinates, and multigroup cross sections to represent the continuous space, direction, and energy variables). A limited evaluation of neutron dpa rates at the HB-2 nozzle corner region using a Monte Carlo model was performed in [3]. The first application of a hybrid Monte Carlo/discrete ordinates methodology to a HFIR dpa rate analysis [4] applied the Consistent Adjoint Driven Importance Sampling (CADIS) methodology [5] to evaluate the dpa rates in the vicinity of HB-2, where dpa rates in the vessel are highest due to the effects of neutron streaming down the enlarged beam tube. In the CADIS method, which was developed to accelerate the statistical convergence of a single Monte Carlo tally, an adjoint deterministic calculation is used to generate particle importances in space and energy. Monte Carlo target weights and a consistently biased Monte Carlo source are then constructed from the importance values. The adjoint source spectrum for the deterministic calculation is based on the response function of interest for the final Monte Carlo simulation.

The FW-CADIS method [6] is used when more extensive Monte Carlo tally results, including mesh tallies, are required. In the FW-CADIS approach, a deterministic forward calculation is first used to generate an estimate of the flux or response of interest throughout the model phase space. An adjoint source corresponding to the Monte Carlo tallies of interest is then constructed based on the deterministic forward solution, with the adjoint source magnitude in each cell being the inverse of the response, and the weight window target values and biased source are determined in the same way as in the CADIS method. Details of the FW-CADIS method can be found in the references. In this study we apply the FW-CADIS technique in the ADVANTG code [7] to develop weight windows and consistently biased sources for use in the MCNP radiation transport code [8]. We then use MCNP to calculate neutron and photon dpa rates in the "standard" dosimetry locations and subsequently for an axial section of the entire reactor vessel using a detailed cylindrical mesh tally.

\section{MCNP and ADVANTG Calculations}

The MCNP model we used for the HFIR dpa analyses has been developed over several years for a range of applications, most of which are focused on the core and reflector regions. For dpa calculations we added details for the nozzles (where the beamlines penetrate the reactor vessel) and for Charpy V-notch (CVN) surveillance capsules and neutron and photon dosimeters. The CVN capsules are located in "keys" in the vicinity of each beamline, and in the "carousel" around HB-2. There are also five tubular dosimeters near HB-2. A cross-sectional view of the model at the core axial midplane $(\mathrm{z}=0)$ is shown in Fig. 1.

Because the ADVANTG sequence generates an MCNP source definition that is biased in space and energy, we ran MCNP in fixed-source (rather than eigenvalue) mode for our dpa calculations. We first generated a spatially dependent fixed source from an eigenvalue calculation by accumulating fission neutron production sites in a cylindrical mesh tally which consists of 19 axial zones and 17 radial zones ( 8 for the inner fuel and 9 for the outer fuel). These axial and radial zones are consistent with the fuel composition modeling that is employed in the model. Our eigenvalue calculation included 8000 active cycles with 100,000 histories per cycle. This resulted in relative errors of less than $0.1 \%$ for the fission neutron production rate in almost all of the 323 fuel zones. We then converted the mesh tally data into an equivalent MCNP spatial distribution for the fixed-source definition. We applied the Watt spectrum for thermal neutron fission in U-235 for the energy distribution of the fission neutrons. We note that this approach ignores the effect of different energy spectra for fission from other isotopes, but it is appropriate for this highly enriched uranium (HEU) core and is superior to collecting an energy-dependent tally of 


\section{$15^{\text {th }}$ ISRD}

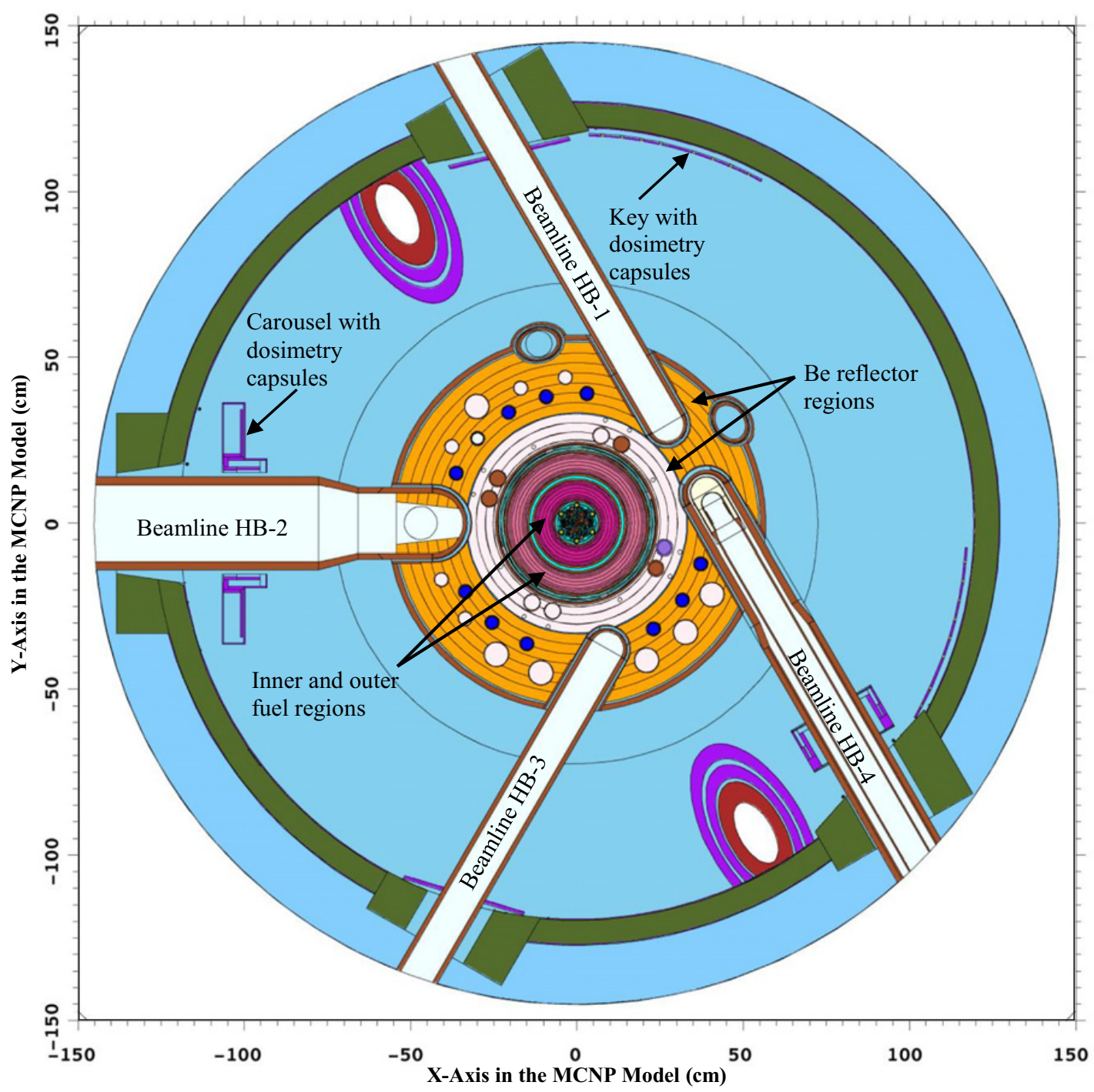

Figure 1. Cross-sectional view of the HFIR MCNP model at the core axial midplane. The core consists of two annular fuel regions around a central flux trap. Inner and outer beryllium reflectors surround the fuel. The flux trap and reflectors contain experimental locations that are used for isotope production and material irradiations.

the fission neutron production. For deep-penetration shielding applications such as this, the high-energy portion of the fission neutron spectrum can have a significant impact on the response of interest, and it is difficult to obtain a well-converged high-energy source term from a tallying approach such as this.

Using the FW-CADIS methodology described in the previous section, we generated weight windows and a consistently biased source using the following parameters: a spatial mesh of $122 \times 122 \times 83$, the SCALE 27n19g coupled multigroup cross-section library [9], and a quadruple range (QR) quadrature set [10] with 12 polar angles and a triangular arrangement of azimuthal angles. We applied the response weighting option in ADVANTG, where the response of interest for each Monte Carlo tally is based on folding the neutron and photon fluxes with dpa cross sections. For neutron dpa rates we applied the ASTM Standard E693-12 [11]. For gamma dpa rates we used iron displacement cross sections 


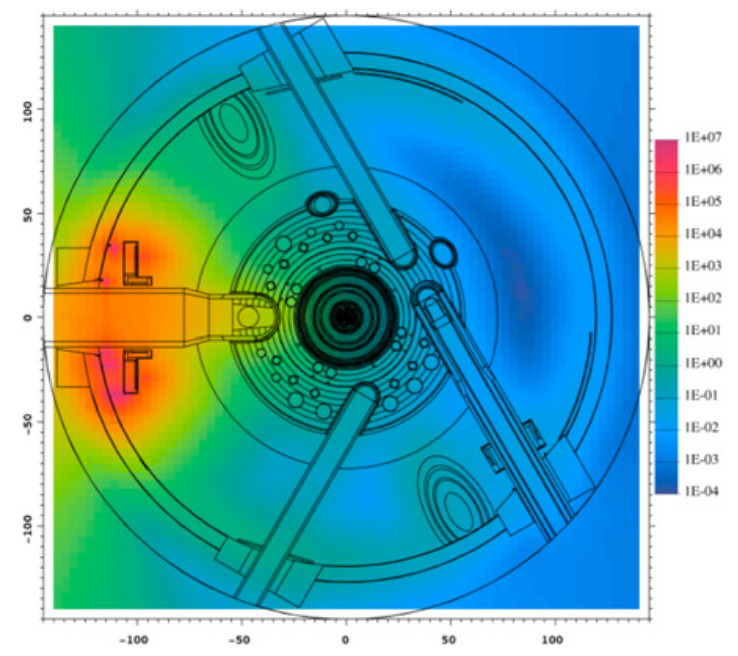

Figure 2. Adjoint fluxes generated by ADVANTG for neutrons in the energy range from 1.827-3.012 MeV at the axial midplane of the HFIR core. The adjoint source regions were the dosimetry locations in the vicinity of HB-2.

from [12]. This combination of spatial mesh and energy groups produces weight window files that are approximately $667 \mathrm{MB}$ in size. We also generated weight windows using the BUGLE library [13], which has 47 neutron groups and 20 gamma groups. This results in weight window files that are approximately $50 \%$ larger than those using the SCALE 27/19 library. There was no significant difference in the MCNP results when we used BUGLE. We chose to use the 27/19 library because of the smaller weight window size, as we have occasionally observed difficulties when using large weight window files with parallel MCNP calculations. A very recent addition to the ADVANTG code provides the ability to collapse an importance map by a specified fraction, which allows the user to apply more detailed cross-section libraries and spatial meshes for the forward and adjoint deterministic calculations, and results in smaller weight window files. We have not yet applied that option to the HFIR vessel damage analysis.

Figures 2 and 3 illustrate the adjoint fluxes at the core midplane elevation for neutron group 3 (1.827-3.012 MeV) for two cases. In Fig. 2 the adjoint source is based on MCNP tallies in the vicinity of the HB-2 nozzle and in the dosimetry capsule carousel that is located around the beamline. In Fig. 3 the adjoint source is based on the dosimetry capsules located in the vicinity of each beamline nozzle and in the keys near HB-1 and HB-4. The neutron target weights for this energy range are proportional to the inverse of the adjoint fluxes. The differences in the adjoint fluxes for these two cases illustrate the significant range of target weights. Clearly, generating useful target weights with this level of detail would be extremely challenging without the use of FW-CADIS.

After generating the weight windows and biased sources with ADVANTG, we ran MCNP to estimate the dpa rates at the locations of interest. We used MCNP 5.160 on a 512-core Linux cluster. For our first set of calculations (dpa rates at the keys, dosimetry locations, and corner/annulus/weld locations) we ran $4 \times 10^{9}$ histories. MCNP run times were about $2500 \mathrm{CPU}$-hours. Tally relative errors at the locations of interest were all less than $2 \%$ at the one-sigma level, and most were less than $1 \%$. We calculated dpa rates for beginning-of-cycle (BOC) and end-of-cycle (EOC) conditions.

The more accurate geometry modeling provided by the MCNP analysis also allowed us to evaluate a number of effects that could not be readily determined from the earlier combination of $2 \mathrm{D}$ and $3 \mathrm{D}$ deterministic analyses. For instance, our calculations of the dpa rates in the dosimetry capsules located in the two carousel rings around HB-2 indicated that there is a lateral asymmetry, with the dpa rates being slightly higher in the $-\mathrm{Y}$ direction (as shown in Fig. 1) than the $+\mathrm{Y}$ direction at a given elevation. 


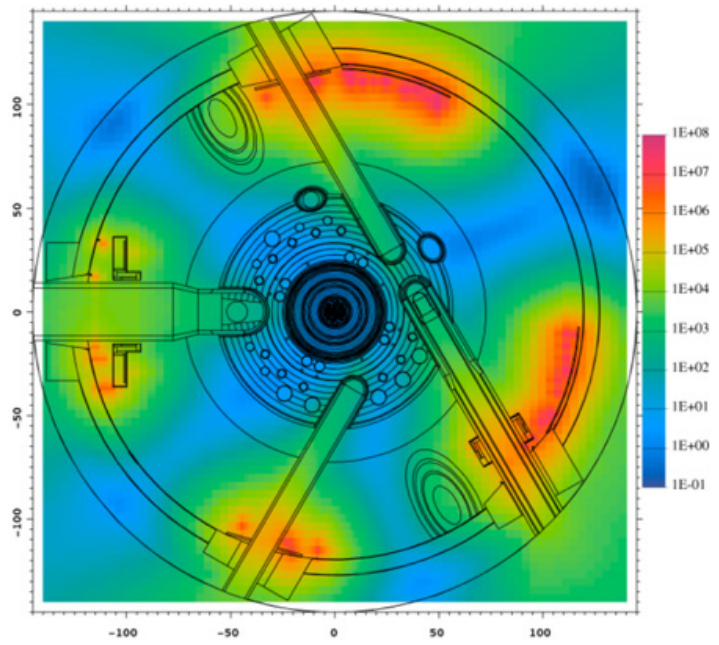

Figure 3. Adjoint fluxes generated by ADVANTG for neutrons in the energy range from 1.827-3.012 MeV at the axial midplane of the HFIR core. The adjoint source regions were the dosimetry locations in the vicinity of all four beamlines.

Using a few ADVANTG/MCNP calculations, we were readily able to confirm that this effect was a result of variations in material compositions in target regions in the beryllium reflector around the inner end of the HB-2 beamline. We also ran a series of calculations in which we assessed potential shifts in the location of the carousel around HB-2. It would not be feasible to obtain the geometric detail necessary for that evaluation using a discrete ordinates analysis, and it would be difficult to obtain Monte Carlo results that are converged well enough to assess the effect of a minor carousel displacement without the use of FW-CADIS.

After demonstrating the effectiveness of ADVANTG in developing variance reduction parameters for all of the beamline dosimetry locations, we applied a cylindrical mesh tally to calculate the dpa rates averaged over the first 1/8 in. of the reactor vessel base metal. We divided this annular region into axial zones of 1-in. height over the interval from -21.5 in. to 21.5 in. and into 360 uniform azimuthal bins, for a total of 15,480 mesh cells. Note that some of those tally cells occur in regions around the nozzles that are not part of the reactor vessel itself (e.g., the beamline walls and the water regions between the beamlines and the nozzles). Figure 4 illustrates the EOC neutron and photon dpa rates around the circumference of the reactor vessel at the core midplane elevation, including the pronounced peaking of the neutron dpa level around the HB-2 beamline. We have shaded the "non-vessel" tally data to indicate those regions in which the calculated dpa rates do not actually represent the dpa rate in the inner layer of the reactor vessel base metal. Figure 5 illustrates the calculated EOC neutron dpa rates over a portion of the reactor vessel that includes the penetrations for HB-1, HB-2, and HB-3. Figures 6 and 7 show the neutron dpa rates and their relative errors for the quadrant of the reactor vessel that extends from the centerline of HB-2 (the negative X-axis in Fig. 1) to the location of the negative Y-axis in Fig. 1. The cross-hatched regions of those plots indicate locations where the mesh tally result is in one of the beamlines or in the water between the beamline and the reactor vessel. Note that the relative errors are less than $1 \%$ in most locations, including around the perimeter of the HB-2 beamline, where the highest dpa rates occur.

We also used a segmented surface tally to compute the dpa rates around the vessel perimeter at several elevations to compare to the mesh tally results. The axial extents of these segmented surface tallies were $1 \mathrm{in}$., consistent with the mesh tally cells. The purpose of the surface tallies was to compare 


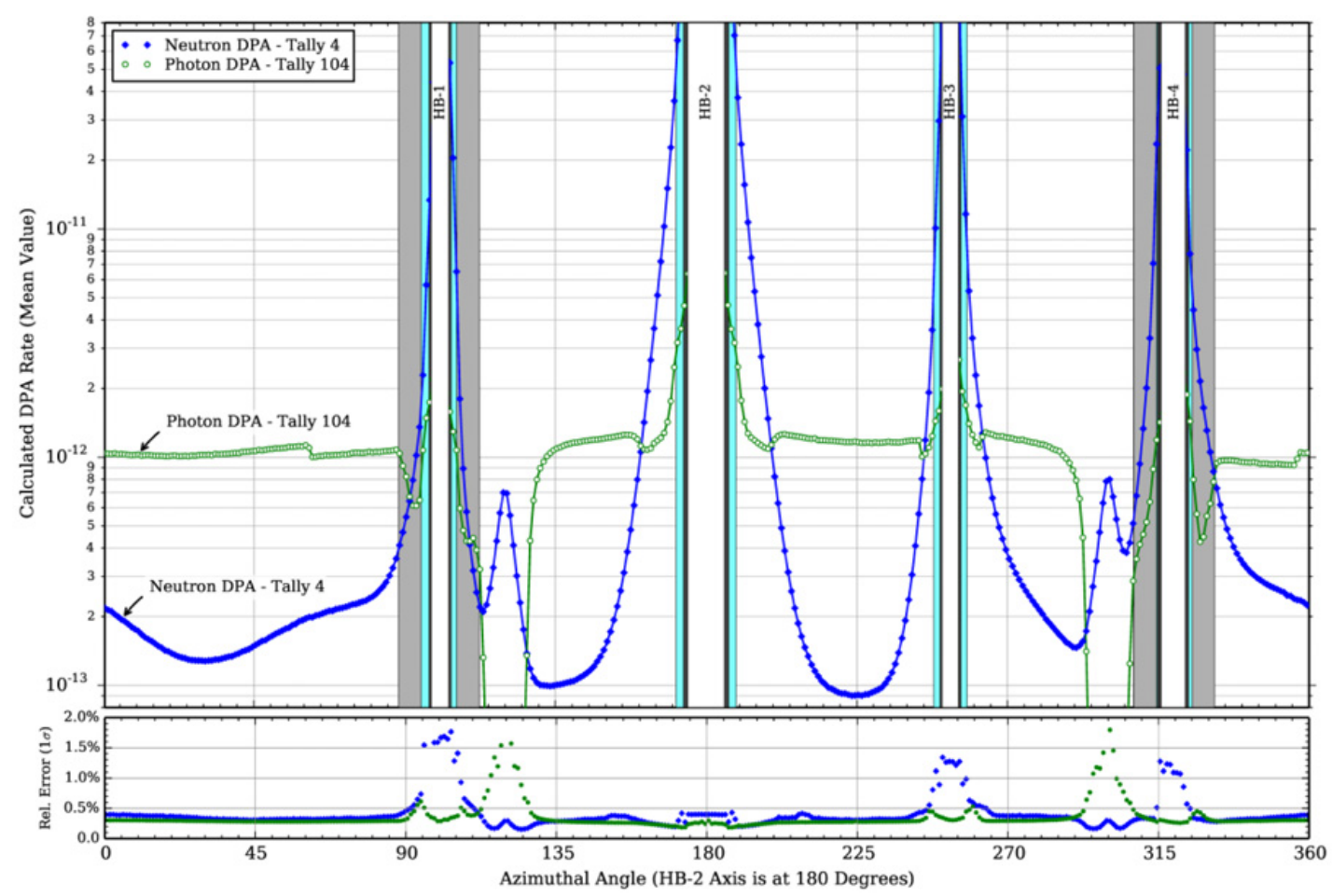

Figure 4. Neutron and photon dpa rates and their relative errors around the reactor vessel perimeter at the elevation of the core midplane. The shaded regions indicate the beamline walls, water between the beamlines and the reactor vessel, and, for HB-1 and HB-4, the protrusion of the nozzle "bosses" inboard of the reactor vessel.

the dpa rates at the clad/base metal interface (the location of the surface tally) with the dpa rates from the mesh tally, which, as noted above, represent an average value over the first 1/8 in. of the base metal. For neutron dpa rates there is essentially no difference between the surface tally and mesh tally results. For photon dpa rates the mesh tally results are reduced by about $5 \%$ relative to the corresponding surface tally values. These differences are consistent with the attenuation expected based on the neutron and photon cross-section data for iron.

\section{Summary and Conclusions}

We have applied the ADVANTG code, which uses the CADIS and FW-CADIS hybrid transport methods to generate variance reduction parameters for MCNP models, to the calculation of dpa rates for HFIR. Our initial evaluation focused on a set of specific model locations that correspond to the positions of surveillance capsules and dosimeters in the vicinity of the four beamlines. Based on the successful application of ADVANTG for those evaluations, we extended our analysis to calculate the dpa rates using a detailed cylindrical mesh tally that includes over 15,000 mesh cells covering the entire perimeter of the reactor vessel over an axial range that extends beyond the top and bottom of the core. Using ADVANTG we were able to create a well-converged detailed map of the neutron and photon dpa rates in the reactor vessel.

We are currently applying the FW-CADIS method to improve isotopic production evaluations for target locations in the HFIR flux trap and reflector regions. While these are not regions of deep 


\section{$15^{\text {th }}$ ISRD}

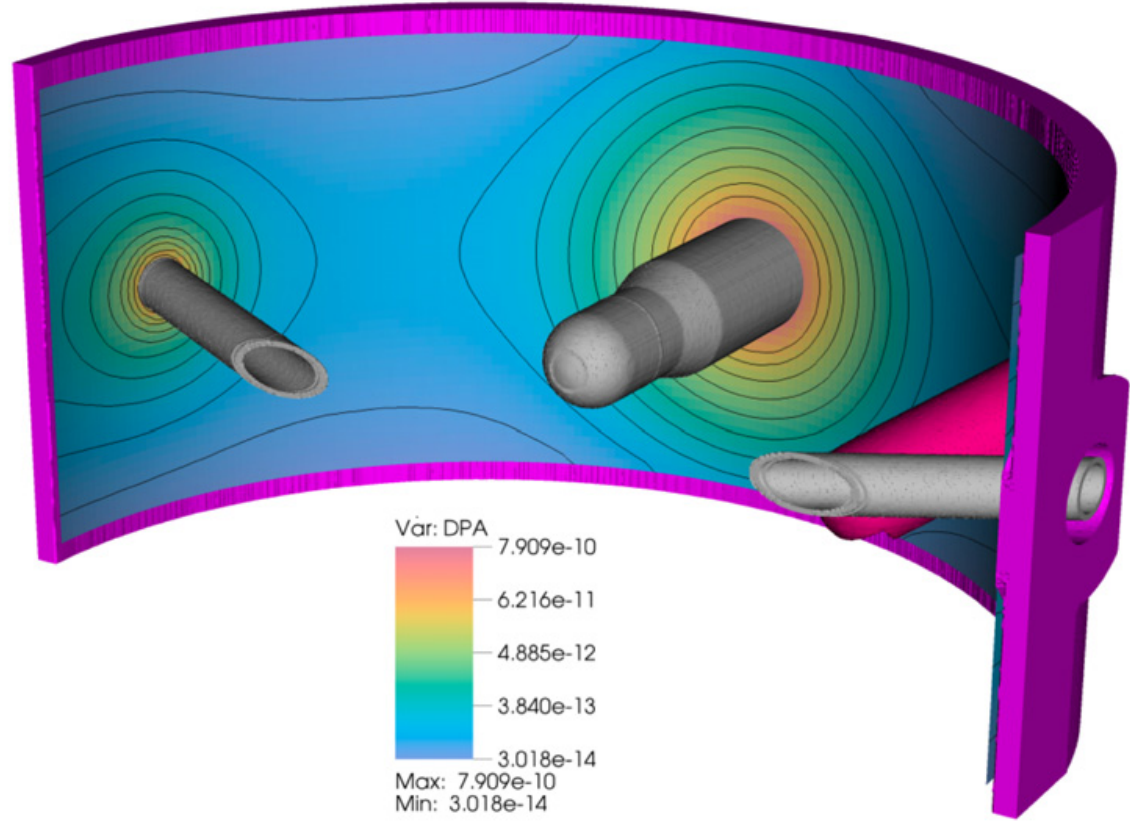

Figure 5. Neutron dpa rates over a portion of the HFIR reactor vessel. The cylindrical gray regions represent HB-1 (to the right), HB-2 (center), and HB-3. The contour line levels are equivalent to those in Figure 6. Only the first three contours are labeled.

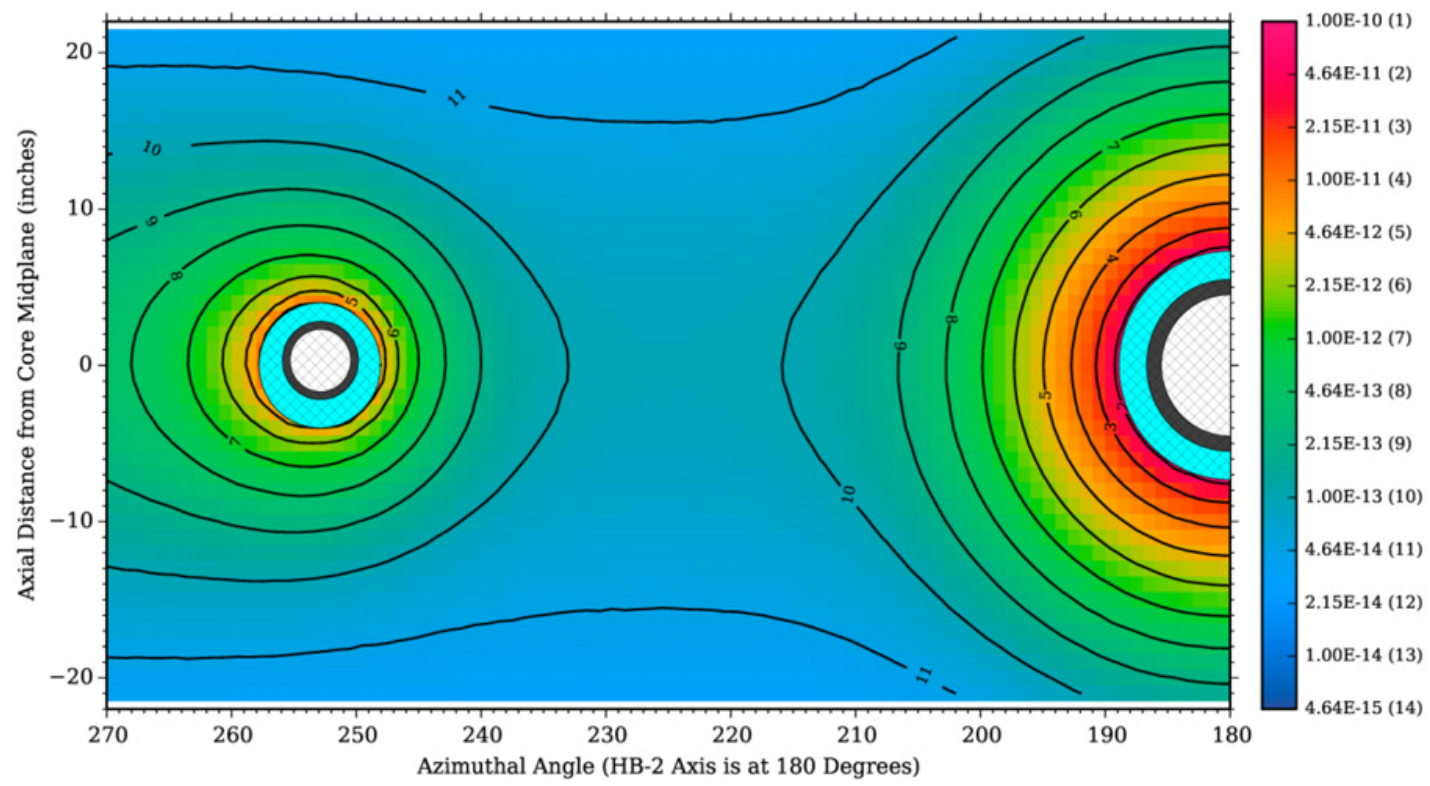

Figure 6. Neutron dpa rates averaged over the first $1 / 8$ in. of the HFIR reactor vessel for the quadrant beginning at the centerline of the HB-2 beamline and extending past the HB-3 beamline. The cross-hatched circular regions indicate those regions around the HB-2 and HB-3 beamlines that are not a part of the reactor vessel. 


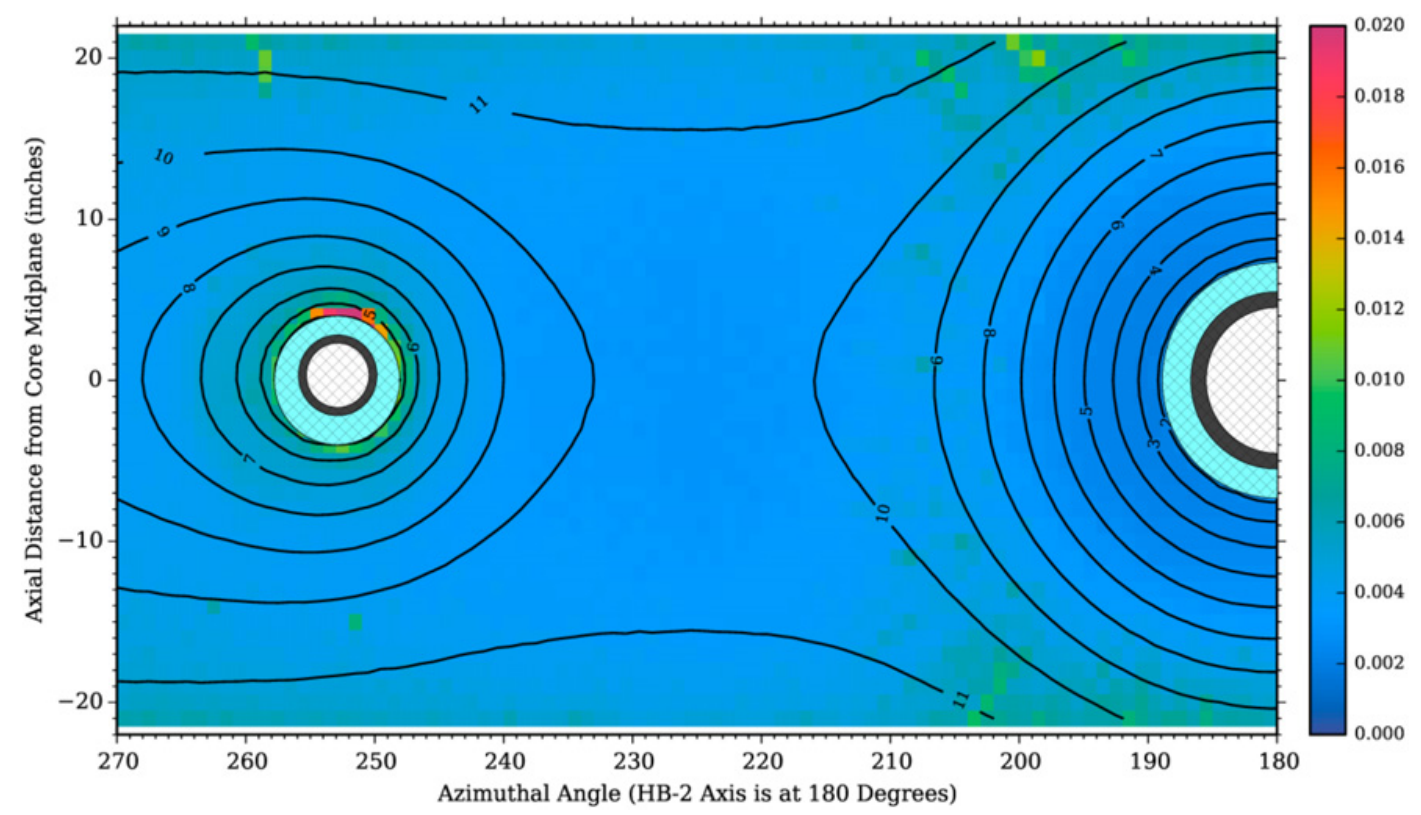

Figure 7. Relative errors at the one-sigma level for the neutron dpa rates shown in Fig. 6. The cross-hatched circular regions indicate those regions around the HB-2 and HB-3 beamlines that are not a part of the reactor vessel. The integer labels on the contour lines correspond to the scale shown in Fig. 6.

penetration shielding (like the reactor vessel), they represent a challenging problem due to the need to obtain accurate predictions of isotopic generation rates for a wide range of nuclides. Without an effective set of weight windows and a consistently biased source, it is difficult to obtain well-converged Monte Carlo neutron fluxes at the high energies that drive some threshold reactions. We are also applying FWCADIS to an evaluation of dpa rates for a low-enriched uranium (LEU) core design and will be assessing the impact of an LEU core on neutron flux levels in the beamlines.

\section{References}

[1] E. D. Blakeman, Neutron and Gamma Fluxes and dpa Rates for the HFIR Vessel Beltline Region (Present and Upgrade Designs), ORNL/TM-13693, Oak Ridge National Laboratory (2000)

[2] E. D. Blakeman and R. D. Cheverton, Neutron and Gamma Fluxes and dpa Rates for the HFIR Vessel Beltline Region (Present and Upgrade Designs) Supplement 2, ORNL/TM-13693/S2, Oak Ridge National Laboratory (2010)

[3] E. D. Blakeman, J. A. Bucholz, and R. D. Cheverton, Calculation of dpa Rates at the HFIR HB-2 Beam Tube Nozzle Corner Using Three-Dimensional Discrete Ordinates and Monte Carlo Models, ORNL/TM-2003/213, Oak Ridge National Laboratory (2004)

[4] E. D. Blakeman, "HFIR dpa-Rate Calculations Using Hybrid Monte Carlo/Discrete Ordinates," Radiation Protection and Shielding Division 2010 Topical Meeting, Las Vegas, NV, April 18-23, 2010

[5] J. C. Wagner and A. Haghighat, "Automated Variance Reduction of Monte Carlo Shielding Calculations Using Discrete Ordinates Adjoint Function," Nucl. Sci. Eng., 128, 186 (1998)

[6] J. C. Wagner, D. E. Peplow, and S. W. Mosher, "FW-CADIS Method for Global and Regional Variance Reduction of Monte Carlo Radiation Transport Calculations," Nucl. Sci. Eng., 176, 37 (2014) 


$$
15^{\text {th }} \text { ISRD }
$$

[7] S. W. Mosher et al., ADVANTG - An Automated Variance Reduction Parameter Generator, ORNL/TM-2013/416, Oak Ridge National Laboratory (2013)

[8] X-5 Monte Carlo Team, "MCNP - A General Monte Carlo N-Particle Transport Code, Version 5. Volume I: Overview and Theory," LA-UR-03-1987, Los Alamos National Laboratory (2003)

[9] D. Wiarda, M. E. Dunn, D. E. Peplow, T. M. Miller, and H. Akkurt, Development and Testing of ENDF/B-VI.8 and ENDF/B-VII.O Coupled Neutron-Gamma Libraries for SCALE 6, ORNL/TM2008/047, NUREG/CR-6990, Oak Ridge National Laboratory (2008)

[10] I. K. Abu-Shumays, "Angular Quadratures for Improved Transport Computations," Transp. Theory Stat. Phys. 30, 169-205 (2001)

[11] ASTM Standard E693-12, "Standard Practice for Characterizing Neutron Exposures in Iron and Low Alloy Steels in Terms of Displacements Per Atom (DPA)," E 706(ID), June 2012

[12] N. P. Baumann, "Gamma-ray Induced displacements in D2O Reactors," Proc. Of the Seventh ASTMO-EURATOM Symposium on Reactor Dosimetry, Strasbourg, France, August 27-31, 1990

[13] J. E. White et al., Production and Testing of the Revised VITAMIN-B6 Fine-Group and the BUGLE-96 Broad-Group Neutron/Photon Cross-Section Libraries Derived from ENDF/B-VI.3 Nuclear Data, ORNL-6795, NUREG/CR-6214, Revision 1 (1995) 